This item was submitted to Loughborough's Research Repository by the author.

Items in Figshare are protected by copyright, with all rights reserved, unless otherwise indicated.

\title{
A Fourier-series-based virtual fields method for the identification of 2-D stiffness and traction distributions
}

PLEASE CITE THE PUBLISHED VERSION

http://dx.doi.org/10.1111/str.12105

PUBLISHER

(c) Wiley

VERSION

AM (Accepted Manuscript)

\section{PUBLISHER STATEMENT}

This work is made available according to the conditions of the Creative Commons Attribution-NonCommercialNoDerivatives 4.0 International (CC BY-NC-ND 4.0) licence. Full details of this licence are available at: https://creativecommons.org/licenses/by-nc-nd/4.0/

\section{LICENCE}

CC BY-NC-ND 4.0

\section{REPOSITORY RECORD}

Nguyen, Truong Tho, Jonathan M. Huntley, lan A. Ashcroft, Pablo D. Ruiz, and Fabrice Pierron. 2019. "A Fourier-series-based Virtual Fields Method for the Identification of 2-D Stiffness and Traction Distributions". figshare. https://hdl.handle.net/2134/21066. 


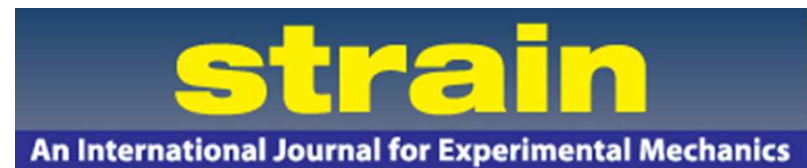

\section{A Fourier-series-based Virtual Fields Method for the identification of 2-D stiffness and traction distributions}

\begin{tabular}{|r|l|}
\hline Journal: & Strain \\
\hline Manuscript ID: & STRAIN-0932.R1 \\
\hline Manuscript Type: & Full Paper \\
\hline Date Submitted by the Author: & $17-$ Jul-2014 \\
\hline Complete List of Authors: & $\begin{array}{l}\text { Nguyen, Tho; Loughborough University, Mechanical and Manufacturing } \\
\text { Engineering } \\
\text { Huntley, Jonathan; Loughborough University, Mechanical and } \\
\text { Manufacturing Engineering } \\
\text { Ashcroft, Ian; University of Nottingham, Faculty of Engineering } \\
\text { Ruiz, Pablo; Loughborough University, Mechanical and Manufacturing } \\
\text { Engineering; } \\
\text { Pierron, Fabrice; University of Southampton, Faculty of Engineering and } \\
\text { the Environment }\end{array}$ \\
\hline Keywords: & $\begin{array}{l}\text { Stiffness identification, Virtual fields method, Fourier series, Unknown } \\
\text { boundary conditions }\end{array}$ \\
\hline & \\
\hline
\end{tabular}


A Fourier-series-based Virtual Fields Method for the identification of 2-D stiffness and traction distributions

\author{
T. T. Nguyen ${ }^{1}$, J. M. Huntley ${ }^{1}$, I. A. Ashcroft ${ }^{2}$, P. D. Ruiz ${ }^{1}$ and F. Pierron ${ }^{3}$ \\ ${ }^{1}$ Loughborough University, Wolfson School of Mechanical and Manufacturing Engineering, \\ Loughborough LE11 3TU, UK \\ ${ }^{2}$ University of Nottingham, Faculty of Engineering, Nottingham NG7 2RD, UK \\ ${ }^{3}$ University of Southampton, Faculty of Engineering and the Environment, Highfield, \\ Southampton SO17 1BJ, UK
}

\begin{abstract}
The Virtual Fields Method (VFM) allows spatial distributions of material properties to be calculated from experimentally-determined strain fields. A numerically-efficient Fourierseries-based extension to the VFM (the F-VFM) has recently been developed, in which the unknown stiffness distribution is parameterised in the spatial frequency domain rather than in the spatial domain as used in the classical VFM. However, the boundary conditions for the FVFM are assumed to be well-defined, whereas in practice the traction distributions on the perimeter of the region of interest are rarely known to any degree of accuracy. In the current paper we therefore consider how the F-VFM theory can be extended to deal with the case of unknown boundary conditions. Three different approaches are proposed; their ability to reconstruct normalised stiffness distributions and traction distributions around the perimeter from noisy input strain fields is assessed through simulations based on a forward finite element analysis. Finally a practical example is given involving experimental strain fields from a diametral compression test on an aluminium disc.
\end{abstract}

Keywords: stiffness identification, virtual fields method, Fourier series, unknown boundary conditions. 


\section{Introduction}

In solid mechanics, robust identification of material properties from experimental data depends on the accuracy of the mathematical model being used, including the magnitudes and positions of the boundary conditions applied to the perimeter of the domain of interest. Despite the importance of the determination of such boundary conditions in inverse engineering problems, only a modest amount of research has been carried out in this area: it is not easy to estimate the traction distributions using pointwise measuring devices, such as strain gauges, attached to the surface of the specimen, particularly in the case of (soft) biological materials.

There exist a number of methods which address the inverse identification of stiffness or of modulus in the literature. A significant amount of research has been carried out to solve for the modulus analytically while exploiting the information from elastography [1] for example. The modulus of incompressible isotropic plane stress problems was also investigated using a variational method whilst weighting by the adjoint operator [2]. An alternative technique to solve for inverse problems of this type is through finite element model updating (or FEMU), which adjusts the unknown quantities through a process to minimise the difference between the response of a numerical model and that measured from experiments. Experimental data used in FEMU can be either static [3] or dynamic [4]. An insightful review of the FEMU together with several other inverse techniques can be found in [5]. The virtual fields method (VFM) is another technique to reconstruct the modulus information within materials with less computational effort than for other techniques. The VFM makes use of an appropriate choice of virtual fields in the equation of the principle of virtual work [6].

Regarding the characterisation of unknown boundary condition distributions in statics, i.e. tractions, displacements, heat fluxes or temperatures on the boundary of the domain of interest, a number of methods have been proposed in the literature. The first is the analytical method, which solves the equilibrium equations of the inverse problems through several mathematical implementations. Typical example of this method can be found in [7]. Many other researchers were successful at determining the distributions of the boundary conditions based on redundant data measured on a portion of the boundary (known as an over-specified boundary). A system of equations which contains parameters from both unknown and overspecified boundaries is established, which can then return the parameters characterising the unknown part of the boundary either iteratively $[8,9]$ or by a single computation [10]. Recent efforts to solving Cauchy boundary problems can be found in [11]. Attempts have also been 
made to identify unknown boundary conditions in dynamics [12]. Inverse problems of this type are typically ill-conditioned [7]. A regularisation method may therefore be needed to stabilise the final results by preventing amplification of measurement errors [8], especially when the noise is significant [12].

In a paper closely related to the aims of the current one, the unknown boundary conditions problem in 3-D was considered by Avril et al. [13] using a finite-element-based VFM. In this case the work done by the unknown tractions on the outer surfaces of a 3-D volume were nulled by using a set of virtual displacement fields that are zero on the boundary. This approach is in fact the basis of the so-called 'windowed traction' technique which will be described later in this paper. Pierron et al. [14] used the VFM with a sinusoidal window function on a dynamic data set of a phantom to determine its shear modulus. Another example is the development of a mechanical consistency indicator to locate impact damage on composite plates [15]. A detailed theory of the VFM can be found in [6].

In view of the limited number of techniques in the literature which are able to simultaneously identify stiffness distributions and unknown boundary conditions, the present paper investigates the unknown traction boundary condition problem as part of the inverse identification of a 2-D stiffness distribution. The approach is an extension to the recently proposed Fourier-series-based virtual fields method (F-VFM) [16] in which the stiffness distribution is parameterised in the spatial frequency domain, rather than the spatial domain as in the classical VFM. Particular advantages of this approach include the ability to control directly the spatial resolution of the reconstructed stiffness distributions, and the existence of a fast algorithm that reduces computational effort by typically 3-4 orders of magnitude. However, the tractions round the boundary are assumed to be known in this first implementation, which seriously limits its applicability to the analysis of many experimental datasets.

The theory underlying the F-VFM is summarised for completeness in Section 2. Three different extensions to the F-VFM, namely the 'experimental traction', the 'windowed traction' and the 'Fourier-series traction' approaches, are then proposed in Section 3 to cope with the situation where boundary information is lacking. Validation of the F-VFM with numerical strain data from a forward finite element analysis is presented in Section 4 followed by its application to real experimental data. Discussion on and conclusions of the new method is found in the last section. 


\section{Summary of the Fourier Virtual Fields Method in 2-D}

The fundamental equation of the VFM comes from the principle of virtual work (PVW) equation (integral form) written for a deformable body, which describes the balance between the virtual works of internal and external forces with any continuous and differentiable virtual displacement field (and its associated virtual strain field). In the case of a quasi-statically loaded 2-D thin structure with negligible body forces and linear elastic constitutive behaviour, the equilibrium equation of the structure can be written as

$$
-\int_{S} \boldsymbol{\epsilon}^{*} \mathbf{Q} \boldsymbol{\epsilon} d S+\int_{\ell} \mathbf{T} \mathbf{u}^{*} d \ell=0
$$

where $\mathbf{Q}$ is the stiffness matrix; $\boldsymbol{\epsilon}$ is the measured strain field vector $\left(\epsilon_{x x}, \epsilon_{y y}, \epsilon_{s s}\right)$, with $\epsilon_{x x}$ and $\epsilon_{y y}$ the normal strains along the $x$ - and $y$-axes of a Cartesian coordinate system and $\epsilon_{s s}$ the engineering shear strain within a domain of interest $(S) ; \mathbf{T}=\left(T_{x}, T_{y}\right)$ is the traction vector acting on a portion $(\ell)$ of the boundary; and $\mathbf{u}^{*}=\left(u_{x}^{*}, u_{y}^{*}\right)$ and $\boldsymbol{\epsilon}^{*}=\left(\epsilon_{x x}^{*}, \epsilon_{y y}^{*}, \epsilon_{s S}^{*}\right)$ are vectors of virtual displacement and virtual strain fields, respectively.

For the case of an isotropic material, Eqn. (1) can be written

$$
\begin{gathered}
\int_{S}\left(\left(\epsilon_{x x}+v \epsilon_{y y}\right) \epsilon_{x x}^{*}+\left(\epsilon_{y y}+v \epsilon_{x x}\right) \epsilon_{y y}^{*}+\frac{1-v}{2} \epsilon_{s S} \epsilon_{s S}^{*}\right) Q_{x x} d S \\
=\int_{\ell}\left(T_{x} u_{x}^{*}+T_{y} u_{y}^{*}\right) d \ell
\end{gathered}
$$

where the stiffness $Q_{x x}$ is related to the elastic modulus $E$ and the Poisson's ratio $v$ through

$$
Q_{x x}=\frac{E}{1-v^{2}}
$$

The early VFM relied on the assumption that $Q_{x x}$ is uniform over the domain, allowing it to be taken out of the left-hand-side integral in Eqn. (2) and thus to a direct and single computation of the stiffness [17]. However, this assumption is often not valid, in which case a continuous parameterisation of the stiffness distribution as polynomials of the spatial variables can be made. Coefficients of the polynomials can be isolated from the integral, and recovered through the use of multiple virtual fields (see for example [18]). A discrete (piecewise) parameterisation has been proposed and successfully applied in 2-D [19], 3-D [13] and to welds [20-22]. 
In the F-VFM, the stiffness distributions are written as Fourier series of the spatial variables $x$ and $y$. In the simplest form, where $v$ is approximated as a known position-independent constant $^{1}, Q_{x x}$ may be written:

$$
Q_{x x}(x, y)=\sum_{m=0}^{M} \sum_{n=-N}^{N} a_{m, n} c_{m, n}(x, y)+\sum_{m=0}^{M} \sum_{\substack{n=-N \\ m+|n| \neq 0}}^{N} b_{m, n} s_{m, n}(x, y)
$$

where $a_{m, n}, b_{m, n}$ are the Fourier coefficients of the term with spatial frequency components $(m, n) ; c_{m, n}=c_{m, n}(x, y)=\cos 2 \pi\left(\frac{m x}{L_{x}}+\frac{n y}{L_{y}}\right)$ and $s_{m, n}=s_{m, n}(x, y)=\sin 2 \pi\left(\frac{m x}{L_{x}}+\frac{n y}{L_{y}}\right) ; L_{x}$ and $L_{y}$ are the dimensions of the domain $(S)$; and $M, N$ the maximum values of indices $m$ and $n$, respectively. The presence of negative frequency coefficients in Eqn. (4) is explained by the number of aliased high frequencies generated by $m$ and $n$ values lying well below Nyquist frequency for typical strain field resolutions presented in this paper, and was discussed in detail in [16]. Due to the even/odd symmetry of the cosine/sine functions, only one of the two indices $m$ and $n$ needs to take negative values. In this case we have chosen index $m$ to take the values from 0 to $N$ whilst index $n$ runs from $-N$ to $N$. The constraint $m+|n| \neq 0$ ensures that $m$ and $n$ in the sine part of the expansion cannot be zero at the same time, which would otherwise lead to a zero row in the coefficient matrix. In certain cases it may be permissible to omit the negative frequencies, i.e. the lower limit on the summation over $n$ can be 0 rather than $-N$. This is the case for the numerical application of Section 4, where the stiffness distribution contains a limited range of spatial frequencies. The more general case, such as that considered in Section 5, requires both positive and negative spatial frequencies. A lower limit on $n$ of $-N$, as specified in Eqn. (4), was used in this case. The total number of unknown Fourier coefficients in Eqn. (4) is

$$
N_{F}=2(M+1)(2 N+1)-1
$$

for the case where the lower limit on $n$ is $-N$, and

$$
N_{F}=2(M+1)(N+1)-1
$$

when the lower limit is 0 .

\footnotetext{
${ }^{1}$ The assumption that $v$ is a known constant is reasonable in many cases, but where it is not, a second Fourier series expansion of the variable $v Q_{x x}$ can be performed. This leads to a second set of Fourier coefficients that need to be solved for as part of the analysis.
} 
Repeated application of Eqn. (2) with a total of $N_{F}$ different virtual fields yields a set of simultaneous equations that can be written in matrix form as

$$
\mathbf{M X}=\mathbf{Y}
$$

in which

$$
\begin{aligned}
& \mathbf{M}=\left(\begin{array}{ccc}
\int_{S} P^{(1)} d S & \ldots \int_{S} P^{(1)} c_{m, n} d S \ldots & \ldots \int_{S} P^{(1)} s_{m, n} d S \ldots \\
\vdots & \vdots & \vdots \\
\int_{S} P^{\left(N_{F}\right)} d S & \ldots \int_{S} P^{\left(N_{F}\right)} c_{m, n} d S \ldots & \ldots \int_{S} P^{\left(N_{F}\right)} s_{m, n} d S \ldots
\end{array}\right) \\
& \mathbf{X}=\left(\begin{array}{c}
a_{0,0} \\
\vdots \\
a_{m, n} \\
\vdots \\
b_{m, n} \\
\vdots
\end{array}\right) \\
& \mathbf{Y}=\left(\begin{array}{l}
\int_{\ell}\left(T_{x} u_{x}^{*(1)}+T_{y} u_{y}^{*(1)}\right) d \ell \\
\int_{\ell}\left(T_{x} u_{x}^{*\left(N_{F}\right)}+T_{y} u_{y}^{*\left(N_{F}\right)}\right) d \ell
\end{array}\right)
\end{aligned}
$$

and

$$
P^{(j)}(x, y)=\left(\epsilon_{x x}+v \epsilon_{y y}\right) \epsilon_{x x}^{*(j)}+\left(\epsilon_{y y}+v \epsilon_{x x}\right) \epsilon_{y y}^{*(j)}+\frac{1-v}{2} \epsilon_{s s} \epsilon_{s s}^{*(j)}
$$

where superscript $j$ denotes the $j$-th virtual strain or displacement field. As described in [16] $\epsilon_{x x}^{*}$ and $\epsilon_{y y}^{*}$ are chosen from a set of cosine waves of unit amplitude, with spatial frequency components $p=0, \ldots, M ; q=-N, \ldots, N$ (giving $(M+1)(2 N+1)$ independent virtual fields), and a set of corresponding sine waves (in which the trivial case $p=q=0$ is excluded), giving an additional $(M+1)(2 N+1)-1$ fields. The total number of chosen cosine and sine virtual fields will therefore be equal to $N_{F}=2(M+1)(2 N+1)-1$, which is the required number to determine uniquely the unknown Fourier series coefficient vector $\mathbf{Y}$. The $u_{x}^{*}$ and $u_{y}^{*}$ fields are calculated from these chosen $\epsilon_{x x}^{*}$ and $\epsilon_{y y}^{*}$ fields, and the $\epsilon_{s s}^{*}$ field in turn from $u_{x}^{*}$ and $u_{y}^{*}$. Equation (7) is then inverted to solve for the vector $\mathbf{X}$ containing the desired Fourier coefficients $a_{m, n}$ and $b_{m, n}$ that describe the unknown stiffness distribution. 


\section{Adaptation of the F-VFM to unknown boundary conditions problems}

This section considers the case where the traction vector $\mathbf{T}$ is not specified on the boundary and therefore vector $\mathbf{Y}$ in Eqn. (7) cannot be evaluated. Adaptation of the general F-VFM is required; this can be done in (at least) three different ways, which we call the 'experimental traction', the 'windowed traction' and the 'Fourier-series traction' approaches hereafter.

\subsection{The 'experimental traction' approach}

This approach uses the elastic constitutive law of the material, whose spatially-varying coefficients are already going to be estimated as part of the analysis, to convert the measured strains around the boundary into equivalent tractions. The matrix $\mathbf{Y}$ will then be modified from its original formula in Eqn. (10). An attractive feature of this approach is its simplicity: no extra variables from the unknown tractions are needed as they are expressed as a function of the existing (unknown) stiffness Fourier coefficients.

Equilibrium of an arbitrary length element on the boundary, lying at angle $\theta$ to the $y$ axis as shown in Figure 1, requires the edge tractions to balance the internal strains as follows:

$$
\begin{gathered}
T_{n}=\left(\left(\cos ^{2} \theta+v \sin ^{2} \theta\right) \epsilon_{x x}+\left(\sin ^{2} \theta+v \cos ^{2} \theta\right) \epsilon_{y y}+\frac{1-v}{2} \sin 2 \theta \epsilon_{s s}\right) Q_{x x} \\
T_{t}=\left(-\frac{1+v}{2} \sin 2 \theta \epsilon_{x x}-\frac{1+v}{2} \sin 2 \theta \epsilon_{y y}+\frac{1-v}{2} \cos 2 \theta \epsilon_{s s}\right) Q_{x x}
\end{gathered}
$$

in which $T_{n}$ and $T_{t}$ are respectively the normal and tangential traction vectors on a (local) length element. The formulae of Eqn. (12) are derived from the parametric equations of Mohr's circle [23]. Table 1 shows the formulae for $T_{n}$ and $T_{t}$ for the particular values of $\theta$ corresponding to the four edges of the rectangular domain, and the formulae for $T_{x}$ and $T_{y}$ (global) computed from $T_{n}$ and $T_{t}$ (local) through the relation:

$$
\begin{aligned}
& T_{x}=\cos \theta T_{n}-\sin \theta T_{t} \\
& T_{y}=\sin \theta T_{n}+\cos \theta T_{t}
\end{aligned}
$$

Combining Eqns. (4), (12), (13) and (10) allows the $\mathbf{Y}$ vector to be written as

$$
\mathbf{Y}=\mathbf{Y}_{1} \mathbf{X}
$$

where 


$$
\mathbf{Y}_{1}=\left(\begin{array}{ccc}
\int_{\ell} R^{(1)} d \ell & \ldots \int_{\ell} R^{(1)} c_{m, n} d \ell \ldots & \ldots \int_{\ell} R^{(1)} s_{m, n} d \ell \ldots \\
\vdots & \vdots & \vdots \\
\int_{\ell} R^{\left(N_{F}\right)} d \ell & \ldots \int_{\ell} R^{\left(N_{F}\right)} c_{m, n} d \ell \ldots & \ldots \int_{\ell} R^{\left(N_{F}\right)} s_{m, n} d \ell \ldots
\end{array}\right)
$$

and

$$
R^{(j)}(x, y)=T_{x} u_{x}^{*(j)}+T_{y} u_{y}^{*(j)}
$$

Substituting vector $\mathbf{Y}$ in its new form into Eqn. (7) leads to the homogeneous system of equations

$$
\mathbf{M}_{1} \mathbf{X}=\mathbf{0}
$$

with

$$
\mathbf{M}_{1}=\mathbf{M}-\mathbf{Y}_{1}
$$

Equation (17) has the trivial solution $\mathbf{X}=\mathbf{0}$. Normalisation of the stiffness distribution by its dc (i.e., average) term $a_{0,0}$ is however an option to produce a non-trivial solution to the homogeneous system of equations (17) as follows. With such a normalisation (see, e.g. [24]chapter 3), the dc term of the Fourier stiffness solution vector becomes 1. Matrix $\mathbf{M}_{1}$ of size $N_{F} \times N_{F}$ can thus be split up into a column vector $\overline{\mathbf{N}}$ of size $N_{F} \times 1$ and a sub-matrix $\overline{\mathbf{M}}$ of size $N_{F} \times\left(N_{F}-1\right)$. The column vector $\overline{\mathbf{N}}$ is the first column of matrix $\mathbf{M}_{1}$ which contains data corresponding to the unit dc term, and sub-matrix $\overline{\mathbf{M}}$ contains the rest of the data. The column vector $\overline{\mathbf{N}}$ is then brought to the right side of Eqn. (17), resulting in the non-homogeneous and over-determined system of equations

$$
\overline{\mathbf{M}} \overline{\mathbf{X}}=\overline{\mathbf{N}}
$$

whose normalised solution $\overline{\mathbf{X}}$ can be computed in a least-squares sense using e.g. the MoorePenrose inversion algorithm ${ }^{2}$ [25]. The formulae for the elements of the matrices in Eqn. (19) are as follows:

\footnotetext{
${ }^{2}$ The Moore-Penrose inversion is implemented in MATLAB using command pinv.
} 


$$
\overline{\mathbf{M}}=\left(\begin{array}{ccc}
\ldots \int_{S} P^{(1)} c_{m, n} d S-\int_{\ell} R^{(1)} c_{m, n} d \ell \ldots & \ldots \int_{S} P^{(1)} s_{m, n} d S-\int_{\ell} R^{(1)} s_{m, n} d \ell \ldots \\
\vdots & \vdots \\
\ldots \int_{S} P^{\left(N_{F}\right)} c_{m, n} d S-\int_{\ell} R^{\left(N_{F}\right)} c_{m, n} d \ell \ldots & \ldots \int_{S} P^{\left(N_{F}\right)} s_{m, n} d S-\int_{\ell} R^{\left(N_{F}\right)} s_{m, n} d \ell \ldots
\end{array}\right)
$$$$
\overline{\mathbf{N}}=-\left(\begin{array}{c}
\int_{S} P^{(1)} d S-\int_{\ell} R^{(1)} d \ell \\
\vdots \\
\int_{S} P^{\left(N_{F}\right)} d S-\int_{\ell} R^{\left(N_{F}\right)} d \ell
\end{array}\right)
$$

$$
\overline{\mathbf{X}}=\frac{1}{a_{0,0}}\left(\ldots a_{m, n} \ldots \quad \ldots b_{m, n} \ldots\right)^{\prime}
$$

\subsection{The 'windowed traction' approach}

The term 'windowing' in spectral analysis refers to the process of multiplying the data by a function (the window function) that normally decays smoothly towards zero at the ends of the signal so as to reduce 'leakage' from the spectral peaks. The proposed 'windowed traction' approach in the F-VFM also involves applying a smoothly varying function to the virtual displacements, but the reason now is somewhat different. Provided the window function is zero on the entire boundary, the virtual work becomes zero over the boundary regardless of the tractions. Knowledge of the tractions is no longer necessary in the stiffness identification. As with window functions used for spectral analysis, continuity of the function is required to ensure the applicability of Eqn. (1). This approach has previously been used with the classical VFM [13, 14]; a mathematical description adapted for the F-VFM is presented below.

The windowed virtual displacement fields $\hat{u}_{x}^{*}$ and $\hat{u}_{y}^{*}$ are defined by $\hat{u}_{x}^{*}=W(x, y) u_{x}^{*}$ and $\hat{u}_{y}^{*}=W(x, y) u_{y}^{*}$ where $W(x, y)$ is the window function that is chosen to be zero on the boundary. In the case of a rectangular domain it is convenient to define $W$ as a product of two 1-D functions: $W(x, y)=W_{x}(x) W_{y}(y)$, where $W_{x}$ is zero at $x= \pm \frac{L_{x}}{2}$ and $W_{y}$ is zero at $y= \pm \frac{L_{y}}{2}$. The matrix $\mathbf{Y}$ of external virtual work in Eqn. (10) can therefore be written 


$$
\widehat{\mathbf{Y}}=\left(\begin{array}{c}
\int_{\ell}\left(T_{x} \hat{u}_{x}^{*(1)}+T_{y} \hat{u}_{y}^{*(1)}\right) d \ell \\
\vdots \\
\int_{\ell}\left(T_{x} \hat{u}_{x}^{*\left(N_{F}\right)}+T_{y} \hat{u}_{y}^{*\left(N_{F}\right)}\right) d \ell
\end{array}\right)=\mathbf{0}
$$

The matrix equation (7) thus has the homogeneous form

$$
\widehat{\mathbf{M}} \mathbf{X}=\mathbf{0}
$$

with

$$
\widehat{\mathbf{M}}=\left(\begin{array}{ccc}
\int_{S} \hat{P}^{(1)} d S & \ldots \int_{S} \hat{P}^{(1)} c_{m, n} d S \ldots & \ldots \int_{S} \hat{P}^{(1)} s_{m, n} d S \ldots \\
\vdots & \vdots & \vdots \\
\int_{S} \hat{P}^{\left(N_{F}\right)} d S & \ldots \int_{S} \hat{P}^{\left(N_{F}\right)} c_{m, n} d S \ldots & \ldots \int_{S} \hat{P}^{\left(N_{F}\right)} s_{m, n} d S \ldots
\end{array}\right)
$$

where

$$
\hat{P}(x, y)=\left(\epsilon_{x x}+v \epsilon_{y y}\right) \hat{\epsilon}_{x x}^{*}+\left(\epsilon_{y y}+v \epsilon_{x x}\right) \hat{\epsilon}_{y y}^{*}+\frac{1-v}{2} \epsilon_{s s} \hat{\epsilon}_{s S}^{*}
$$

The windowed virtual strains $\hat{\epsilon}_{x x}^{*}, \hat{\epsilon}_{y y}^{*}$ and $\hat{\epsilon}_{s s}^{*}$ are obtained from the corresponding windowed virtual displacements $\hat{u}_{x}^{*}$ and $\hat{u}_{y}^{*}$ by differentiation.

The homogeneous matrix equation (24) can then be solved by implementing the normalisation by the de term $a_{0,0}$ as described in the 'experimental traction' approach above. The over-determined matrix equation after the normalisation is

$$
\widehat{\mathbf{M}} \overline{\mathbf{X}}=\widehat{\overline{\mathbf{N}}}
$$

in which $\widehat{\mathbf{M}}$ is the sub-matrix of the normalised matrix $\widehat{\mathbf{M}}$ in Eqn. (25) formed by removing its first column, and $\widehat{\mathbf{N}}$ the column vector equal to the first column of matrix $\widehat{\mathbf{M}}$. The choice of different window functions will be discussed in Section 4 where the 'windowed traction' approach is applied to a specific case.

\subsection{The 'Fourier-series traction' approach}

In the F-VFM adapted by the 'Fourier-series traction' approach the components $T_{x}$ and $T_{y}$ of the traction vector $\mathbf{T}$ are represented as 1-D Fourier series along the boundary, whose 
coefficients can be determined together with the 2-D Fourier stiffness coefficients through a matrix inversion. The mathematical interpretations of the 'Fourier-series traction' approach are presented in this section.

In the case of a domain without corners (i.e., with no discontinuities in the edge normals) a suitable parameterization is as follows:

$$
\begin{aligned}
& T_{x}(\ell)=\sum_{k=0}^{K} \alpha_{k} \cos 2 \pi \frac{k \ell}{L}+\sum_{k=0}^{K} \beta_{k} \sin 2 \pi \frac{k \ell}{L}=\left(\ldots c_{k}(\ell) \ldots s_{k}(\ell) \ldots\right)\left(\ldots \alpha_{k} \ldots \beta_{k} \ldots\right)^{\prime} \\
& T_{y}(\ell)=\sum_{k=0}^{K} \phi_{k} \cos 2 \pi \frac{k \ell}{L}+\sum_{k=0}^{K} \psi_{k} \sin 2 \pi \frac{k \ell}{L}=\left(\ldots c_{k}(\ell) \ldots s_{k}(\ell) \ldots\right)\left(\ldots \phi_{k} \ldots \psi_{k} \ldots\right)^{\prime}
\end{aligned}
$$

where $\ell$ is the distance along the boundary from an arbitrary starting point on the boundary; $L$ is the perimeter of the domain; $K$ is the maximum spatial frequency measured around the boundary; $\alpha_{k}, \beta_{k}, \phi_{k}$ and $\psi_{k}$ are the Fourier coefficients to be determined as part of the identification; $c_{k}(\ell)=\cos 2 \pi \frac{k \ell}{L}$ and $s_{k}(\ell)=\sin 2 \pi \frac{k \ell}{L}$.

When the domain has corners across which the tractions will be discontinuous, the boundary can be split into sub-boundaries between adjacent corners with an expansion of the form given by Eqn. (28) on each sub-boundary. In the problems considered in Section 4, the domain is rectangular and the following parameterization consisting of eight 1-D Fourier series was therefore used:

$$
\begin{aligned}
T_{x}^{[i]}\left(\ell_{i}\right)=\sum_{k=0}^{K_{i}} \alpha_{k}^{[i]} \cos 2 \pi \frac{k \ell_{i}}{L_{i}}+\sum_{k=1}^{K_{i}} \beta_{k}^{[i]} \sin 2 \pi \frac{k \ell_{i}}{L_{i}} & \\
& =\left(\ldots c_{k}\left(\ell_{i}\right) \ldots s_{k}\left(\ell_{i}\right) \ldots\right)\left(\ldots \alpha_{k}^{[i]} \ldots \beta_{k}^{[i]} \ldots\right)^{\prime} \\
T_{y}^{[i]}\left(\ell_{i}\right)=\sum_{k=0}^{K_{i}} \phi_{k}^{[i]} \cos 2 \pi \frac{k \ell_{i}}{L_{i}}+\sum_{k=1}^{K_{i}} \psi_{k}^{[i]} & \sin 2 \pi \frac{k \ell_{i}}{L_{i}} \\
& =\left(\ldots c_{k}\left(\ell_{i}\right) \ldots s_{k}\left(\ell_{i}\right) \ldots\right)\left(\ldots \phi_{k}^{[i]} \ldots \psi_{k}^{[i]} \ldots\right)^{\prime}
\end{aligned}
$$

where superscript $i$ is the edge index $(i=1, . ., 4) ; \ell_{1}=\ell_{3}=y, \ell_{2}=\ell_{4}=x$; and $L_{i}$ and $K_{i}$ are respectively the length and number of cosine/sine terms for the $i$-th edge. 
The substitution of these traction terms into Eqn. (10) results in a $\mathbf{Y}$ vector that can be decomposed into the product of a coefficient matrix $\widetilde{\mathbf{Y}}$ and an unknown vector of traction coefficients $\widetilde{\mathbf{X}}$ as follows:

$$
\mathbf{Y}=\widetilde{\mathbf{Y}} \widetilde{\mathbf{X}}
$$

where

$$
\begin{gathered}
\widetilde{\mathbf{X}}=\left(\begin{array}{lllll}
\widetilde{\mathbf{X}}_{1}^{\prime} & \widetilde{\mathbf{X}}_{2}^{\prime} & \widetilde{\mathbf{X}}_{3}^{\prime} & \widetilde{\mathbf{X}}_{4}^{\prime}
\end{array}\right)^{\prime} \\
\widetilde{\mathbf{Y}}=\left(\begin{array}{llll}
\widetilde{\mathbf{Y}}_{1} & \widetilde{\mathbf{Y}}_{\mathbf{2}} & \widetilde{\mathbf{Y}}_{3} & \widetilde{\mathbf{Y}}_{4}
\end{array}\right)
\end{gathered}
$$

Sub-vector $\widetilde{\mathbf{X}}_{i}=\left(\ldots \alpha_{k}^{[i]} \ldots \beta_{k}^{[i]} \ldots \phi_{k}^{[i]} \ldots \psi_{k}^{[i]} \ldots\right)^{\prime}$ within vector $\widetilde{\mathbf{X}}$ contains the coefficients of the traction Fourier series applied to edge $i$, and sub-matrix $\widetilde{\mathbf{Y}}_{i}$ is defined by

$\widetilde{\mathbf{Y}}_{i}=$

$$
\left(\begin{array}{cccccc}
\ldots \int_{\ell_{i}} u_{x}^{*(1)} c_{k}\left(\ell_{i}\right) d \ell_{i} & \ldots \int_{\ell_{i}} u_{x}^{*(1)} s_{k}\left(\ell_{i}\right) d \ell_{i} & \ldots \int_{\ell_{i}} u_{y}^{*(1)} c_{k}\left(\ell_{i}\right) d \ell_{i} & \ldots \int_{\ell_{i}} u_{y}^{*(1)} s_{k}\left(\ell_{i}\right) d \ell_{i} & \ldots \\
\vdots & \vdots & \vdots & \vdots & \ldots \\
\ldots \int_{\ell_{i}} u_{x}^{*\left(N_{F}\right)} c_{k}\left(\ell_{i}\right) d \ell_{i} & \ldots \int_{\ell_{i}} u_{x}^{*\left(N_{F}\right)} s_{k}\left(\ell_{i}\right) d \ell_{i} & \ldots \int_{\ell_{i}} u_{y}^{*\left(N_{F}\right)} c_{k}\left(\ell_{i}\right) d \ell_{i} & \ldots \int_{\ell_{i}} u_{y}^{*\left(N_{F}\right)} s_{k}\left(\ell_{i}\right) d \ell_{i} & \ldots
\end{array}\right)
$$

With the Fourier parameterisation of the tractions, the resolution matrix equation (7) now involves not only the unknown vector $\mathbf{X}$ of the Fourier stiffness coefficients but also the unknown vector $\widetilde{\mathbf{X}}$ of the Fourier traction coefficients. By combining coefficient matrices $\mathbf{M}$ and $\widetilde{\mathbf{Y}}$ together into a single matrix $\mathbf{M}^{*}$ and the two unknown vectors $\mathbf{X}$ and $\widetilde{\mathbf{X}}$ into a single column vector $\mathbf{X}^{*}$, the equation may be reformulated in homogeneous form as

$$
\mathbf{M}^{*} \mathbf{X}^{*}=\mathbf{0}
$$

with

$$
\begin{gathered}
\mathbf{M}^{*}=(\mathbf{M} \mid(-\widetilde{\mathbf{Y}})) \\
\mathbf{X}^{*}=\left(\mathbf{X}^{\prime} \mid \widetilde{\mathbf{X}^{\prime}}\right)^{\prime}
\end{gathered}
$$

The normalisation of the equation by the de term $a_{0,0}$ of the Fourier stiffness may be implemented as presented in Section 3.1. The first column of matrix $\mathbf{M}^{*}$, denoted $\overline{\mathbf{N}}^{*}$, can thus be taken to the right hand side of Eqn. (34) leaving sub-matrix $\overline{\mathbf{M}}^{*}$ which contains the rest of the data. The resulting non-homogeneous and over-determined system of equations 


$$
\overline{\mathbf{M}}^{*} \overline{\mathbf{X}}^{*}=\overline{\mathbf{N}}^{*}
$$

can then be solved as described in Section 3.1.

\section{Validation of the adapted F-VFM approaches with numerical data}

Results of the three adapted F-VFM approaches from Section 3 are presented in this section. Numerical strain fields from a forward finite element model were used to validate the algorithms, rather than experimental fields, because of the difficulty in obtaining experimental traction distributions around the boundary. The deformation fields were then fed into the adapted F-VFM equations to recover both the stiffness distribution and the traction profiles.

\subsection{Plate of 'egg-box' stiffness pattern under non-uniform biaxial loads}

The numerical model for this section consisted of a thin square plate of size $L_{x} \times L_{y}=10 \times 10$ $\mathrm{mm}^{2}$ and of thickness $t=1 \mathrm{~mm}$. The traction distributions were applied to the edges of the plate in both horizontal and vertical directions with a stepped stress profile pointing outwards, as shown in Figure 2. The magnitudes of the steps in the stress profile are in the ratio of 1:2, being 0.5 and $1 \mathrm{MPa}$ respectively. The material was chosen to be linear elastic isotropic with the reference elastic modulus distribution of an 'egg-box' pattern defined by:

$$
E^{\mathrm{ref}}=20+\cos 2 \pi\left(\frac{2 x}{L_{x}}+\frac{y}{L_{y}}\right)+\sin 2 \pi\left(\frac{x}{L_{x}}+\frac{2 y}{L_{y}}\right)(\mathrm{MPa})
$$

and a constant Poisson's ratio $v=0.3$. Plane stress conditions are applicable in this case since the plate thickness is relatively small compared to the other dimensions. The plane-stress reference stiffness distribution $Q_{x x}^{\text {ref }}$ is calculated from the reference modulus using Eqn. (3) and the non-dimensional distribution $\bar{Q}_{x x}^{\text {ref }}$ is deduced by dividing by the dc term, i.e.

$$
\bar{Q}_{x x}^{\mathrm{ref}}=\frac{Q_{x x}^{\mathrm{ref}}}{21.98}=1+0.05 \cos 2 \pi\left(\frac{2 x}{L_{x}}+\frac{y}{L_{y}}\right)+0.05 \sin 2 \pi\left(\frac{x}{L_{x}}+\frac{2 y}{L_{y}}\right)
$$

The plate was meshed using $1000 \times 1000$ linear quadrilateral (QUAD4) elements, then analysed by the commercial finite element package MscMarc ${ }^{\mathrm{TM}} 2010$. The three numerical strain components $\epsilon_{x x}, \epsilon_{y y}$ and $\epsilon_{s s}$ provided by the forward analysis were used as the input to 
the adapted F-VFM approaches, with traction information excluded. In what follows, the reconstructed (normalised) stiffness maps are compared to the normalised stiffness $\bar{Q}_{x x}^{\text {ref }}$.

\subsection{Identification of 'egg-box' stiffness distribution and traction profiles by 3 adapted-F- VFM approaches}

Stiffness identification from the 'experimental traction' approach was achieved by computing the normalised solution vector $\overline{\mathbf{X}}$ defined in Eqn. (22), through a matrix inversion of the normalised matrix Eqn. (19). The stiffness map determined by the 'experimental traction' approach is displayed in the top row of Figure 3 with the choice of $M=N=20$ cosine/sine terms in the stiffness Fourier series, resulting in a total of 880 unknown coefficients (excluding the dc term which is 1 due to the normalisation). The reconstructed pattern is disturbed by a number of horizontal and vertical fringes. This artefact consistently occurs at a spatial frequency equal to that of the highest spatial frequency in the modulus expansion; a simple procedure was described in [16] to remove it by convolution with a square kernel of size equal to the pitch $p$ of the fringes, where $p=\frac{L_{x}}{N} \mathrm{~mm}$. One drawback is that any point within a distance $p / 2$ from the edges of the map is influenced by the stiffness values outside the region of interest, which are unknown and here assumed to be zero. This ribbon of low magnitude data is therefore masked out, giving the figures in the middle column of Figure 3.

The effect of different window functions on the stiffness distributions recovered by the 'windowed traction' approach was investigated. The differences were not very significant, so only the results obtained from the use of one of them (the cosine window function $W(x, y)=$ $\left.\cos \frac{\pi x}{L_{x}} \cos \frac{\pi y}{L_{y}}\right)$ are presented, as shown in the middle row of Figure 3. Other potential window functions which can be used in this application include polynomial window function $W(x, y)=\left(x \pm \frac{L_{x}}{2}\right)\left(y \pm \frac{L_{y}}{2}\right)$, Hann window function $W(x, y)=0.5^{2}\left(1 \pm \cos \frac{2 \pi x}{L_{x}}\right)(1 \pm$ $\left.\cos \frac{2 \pi y}{L_{y}}\right)$ or sinc window function $W(x, y)=\operatorname{sinc}\left(\frac{2 \pi x}{L_{x}}\right) \operatorname{sinc}\left(\frac{2 \pi y}{L_{y}}\right)$. More information on how to select appropriate window functions can be found in [26].

The results of the 'Fourier-series traction' approach were achieved with the assumptions that no shear traction component is found on any edge of the plate, and the 4 normal traction distributions are identical, meaning that only a single Fourier series representing the normal traction profile is required. This use of a priori information helps reduce the number of 
variables involved. A value $K=20$ was chosen, leading to a total of 921 unknown coefficients, compared with the 880 variables required by the two other approaches. The identification results from this approach are shown in the bottom row of Figure 3.

Whilst traction profiles of the 'experimental traction' and 'windowed traction' approaches are recovered by using Eqn. (12), those of the 'Fourier-series traction' approach are reconstructed by using Eqn. (28). The traction results are shown in Figure 4 together with the reference traction profile.

\subsection{Noise sensitivity of the F-VFM-adapted approaches}

In order to simulate the effect of the noise on the performance of the F-VFM, the numerical displacement components $u_{x}$ and $u_{y}$ were modified by a number of additive white noise patterns, with standard deviation $\sigma$ ranging from $10^{-3} \mathrm{~mm}$ (low noise level) to $10^{-2} \mathrm{~mm}$ (high noise level). The noise was added to the displacements, rather than the strain fields, as the three strain components are correlated with each other through the displacement-strain relations. For comparison, the ranges of displacements (i.e., minimum to maximum across the full field) were $0.680 \mathrm{~mm}$ and $0.684 \mathrm{~mm}$ for the noise-free $u_{x}$ and $u_{y}$ fields, respectively. For each of ten noise levels, 100 noise patterns were generated and the identification of stiffness distribution was therefore carried out a total of 1000 times.

The derivative $\partial u_{j} / \partial x$ (where $j=x$ or $y$ ) at any given point $(x, y)$ was estimated from the $u_{j}$ values over a square region of size $N_{s} \times N_{s}$ pixels centred on $(x, y)$. This was done by least squares fitting a first order polynomial to the displacement data along each row of the square, and then averaging the $N_{s}$ resulting best-fit gradients over all the rows. The derivative with respect to $y$ was carried out in the same way but with the least squares fitting carried out along the columns of the square and the gradients averaged over the columns. Two gradient kernels of size $7 \times 7$ and $13 \times 13$ pixels were investigated. Both are small compared to the pitch of the highest frequency Fourier coefficient ( $p=50$ pixels) and therefore introduce relatively little additional blurring to the recovered stiffness distribution.

Figure 5 presents the 'egg-box' strain fields with different displacement noise level added. The 'egg-box' stiffness distributions reconstructed from noisy strain data of $\sigma=5 \times 10^{-3} \mathrm{~mm}$ (medium noise level) by 3 approaches are depicted in Figure 6. A more uniform pattern of the stiffness error map is produced by the 'windowed traction' approach, compared to those from the other approaches, with a lower root mean square error $(0.005$ versus 0.031 and 0.007 
from the 'experimental traction' and 'Fourier-series traction' approaches, respectively, at the medium level of noise) (see Figure 6). The corresponding traction distributions determined from the reconstructed stiffness are shown in Figure 7. The windowed traction approach slightly underestimates the $T_{x}$ tractions, and the experimental traction approach slightly overestimates them. In both cases, the unfiltered stiffness distribution was used because the filtered distribution has a missing band around the border as described earlier in section 4.2. The calculated tractions were then smoothed by convolving with a 1-D moving average filter of size 50 pixels. The deviations between the recovered and reference tractions can therefore be seen as due to a combination of edge effects (to which Fourier methods are generally prone) and 1-D spatial smoothing as opposed to the 2-D spatial smoothing as used in the calculation of the stiffness distribution. The closest agreement is given by the Fourier series traction approach which is also the smoothest of the three distributions. The lower noise level is not surprising as the representation of the tractions with a limited range of spatial frequencies has filtering implicitly built in to the method. Figure 8 presents the mean and standard deviation values of the stiffness errors (in MPa) at different gradient kernel sizes and different noise levels. In this figure, the 'known traction' results refer to the situation where the tractions on the boundary of the plate are known (which are actually brought directly from the numerical model of the plate), and the reconstruction of stiffness distributions of the plate with noisy data were undertaken as shown in detail in [16]. As would be expected intuitively, at lower noise levels there is less difference in the stiffness errors when using either the small or large kernel, with the discrepancies in stiffness errors increasing at higher noise levels. At the lower noise level, the mean values of the stiffness error distributions seem to be less sensitive to the kernel sizes, and become more sensitive to kernel size at higher noise levels, as presented by wider error bars.

\section{Application of the adapted F-VFM approaches to experimental data}

In this section, one of the adapted F-VFM approaches, the 'windowed traction' approach, is applied to experimental data from a Brazilian test on a circular aluminium disc. The diameter and thickness of the disc were $36 \mathrm{~mm}$ and $6 \mathrm{~mm}$, respectively. A vertical compressive load of $9 \mathrm{kN}$ was applied to the top and bottom of the disc, as shown in Figure 9. The material is considered elastic isotropic in this case, which can be characterised by its elastic modulus $E$, or stiffness $Q$. The Poisson's ratio was assumed to be known, with a typical reference value 
of 0.3. The in-plane displacement field of the disc was determined by a well-known grid method [27] from which the three in-plane strain components were obtained by differentiation [27]. Only a part of the dataset within a rectangular region of interest (ROI) is used in this application, covering an area of $\sim 25.44 \times 18.24 \mathrm{~mm}^{2}$ (equivalent to $x / D=-$ $0.35 \ldots 0.35$ and $y / D=-0.25 \ldots 0.25)$. Experimental strain fields within the ROI are presented in Figure 10 and compared with the corresponding strain fields computed analytically from Hertzian contact theory [28].

The unknown stiffness distribution of the disc is expanded by a Fourier series as in Eqn. (4) with $M=N=15$ cosine/ sine terms in its representation, resulting in a total of 991 variables by Eqn. (5), or 990 after normalisation of the stiffness by the de term. In this application the material is known to be homogeneous and well-characterised by a single modulus value. Performing the analysis with such a large number of variables, and on data from just a single load case, thus provides a useful test of the numerical stability of the proposed technique in a practical application because any variations in recovered stiffness must be due to the algorithm rather than to the real material behaviour.

As the tractions on the edges of the ROI are unspecified, the stiffness distribution was reconstructed using the 'windowed traction' approach, as presented in Section 3.2. The smoothing was done by convolution with an equally-weighted square mask of size $7 \times 7$ pixels, the side of which equals the pitch of the highest spatial frequency of the reconstructed stiffness distribution. Cross-sections of the calculated normalised stiffness along the top, middle and bottom of the ROI are plotted in Figure 11(a). As would be expected for a sample with constant modulus, these show a normalized stiffness of unity with only small fluctuations (approximately 10.4\% root mean square variation) across the ROI. Experimental tractions distributed over the top and bottom edges of the ROI can then be recovered using the reconstructed stiffness and the input strain fields by Eqns. (12) and (13).

Direct comparison with the analytical traction distributions requires the latter to be normalised by the de term $Q_{0}$ of the reconstructed stiffness distribution. The in-plane stress distribution in a circular disc of radius $R$ according to the Hertz theory (see, e.g. [29]) is

$$
\sigma_{x x}=-\frac{2 P}{\pi D t} f(x, y) ; \quad \sigma_{y y}=-\frac{2 P}{\pi D t} g(x, y) ; \quad \tau_{x y}=\frac{2 P}{\pi D t} h(x, y)
$$

in which $P$ is the compressive load, $D=2 R$ the disc's diameter, $t$ the disc's thickness, and the non-dimensional functions $f, g$ and $h$ are defined by 


$$
\begin{gathered}
f(x, y)=D\left(\frac{x^{2}(R-y)}{\left((R-y)^{2}+x^{2}\right)^{2}}+\frac{x^{2}(R+y)}{\left((R+y)^{2}+x^{2}\right)^{2}}-\frac{1}{2 R}\right) \\
g(x, y)=D\left(\frac{(R-y)^{3}}{\left((R-y)^{2}+x^{2}\right)^{2}}+\frac{(R+y)^{3}}{\left((R+y)^{2}+x^{2}\right)^{2}}-\frac{1}{2 R}\right) \\
h(x, y)=D\left(\frac{x(R-y)^{2}}{\left((R-y)^{2}+x^{2}\right)^{2}}+\frac{x(R+y)^{2}}{\left((R+y)^{2}+x^{2}\right)^{2}}\right)
\end{gathered}
$$

Normalised versions of the analytical traction distributions on the top/bottom edge of the ROI computed from the corresponding stress fields can be written as

$$
\begin{aligned}
& \bar{T}_{x}^{(i)}=\frac{\sigma_{x y}^{(i)}}{Q_{0}}=-\frac{2 P}{Q_{0} \pi D t} h(x, y) \\
& {\overline{T_{y}}}^{(i)}=\frac{\sigma_{y y}^{(i)}}{Q_{0}}=-\frac{2 P}{Q_{0} \pi D t} g(x, y)
\end{aligned}
$$

where superscript $i=1,2$ stands for the top or bottom edge, respectively, of the ROI. The factor $2 P /\left(Q_{0} \pi D t\right)$ in Eqn. (42) is an unknown, but it can be determined from the experimental strain value $\epsilon_{y y}^{(c)}$ at the centre of the ROI through the classical stress-strain relation:

or

$$
\begin{gathered}
\epsilon_{y y}^{(c)}=\frac{1}{E_{0}}\left(\sigma_{y y}^{(c)}-v \sigma_{x x}^{(c)}\right)=-\frac{3+v}{1-v^{2}} \frac{2 P}{Q_{0} \pi D t} \\
\frac{2 P}{Q_{0} \pi D t}=-\frac{1-v^{2}}{3+v} \epsilon_{y y}^{(c)}
\end{gathered}
$$

Subsequently, $\bar{T}_{x}^{(i)}$ and ${\overline{T_{y}}}^{(i)}$ over the top and bottom edges of the ROI (at locations $y / D=$ \pm 0.25 ) are determined using Eqn. (42) and then presented in Figures 11(b) and (c) respectively. The main sources of error in the identification of stiffness and traction distributions might come from the imperfectness of the experiment which generates significant noise in the experimental data, and from the inaccuracy of the analytical Hertz contact model used in the paper when compared with other models e.g. Hondros model which considers distribution of loads at contact surfaces, JKR model which accounts for adhesive contact, etc. Considering the differences between the experimental and analytical input strain fields in Figure 10, the agreement between the analytical and recovered traction distributions can be considered reasonable. 


\section{Conclusions}

Three approaches to extending the F-VFM to deal with the problem of unknown boundary conditions on two-dimensional geometries have been developed. The approaches, which we call the 'experimental traction', the 'windowed traction' and the 'Fourier-series traction' approaches, reconstruct spatial distributions of the stiffness within the domain of interest and of the traction components around its perimeter. In each case, the distributions can only be recovered up to a scale factor of the average stiffness.

Applications of the three approaches to numerical data have been presented, involving a biaxially-loaded sample with a stepped distribution of edge tractions. In the absence of noise, all three approaches resulted in accurate reconstructions of both stiffness and tractions, with identification error from the 'windowed traction' approach far less than those of the two other approaches. Sensitivity analyses of the approaches to noise show a root mean square identification error of $\sim 14 \times, 2.1 \times$, and $1.9 \times$ in recovered stiffness distribution for the 'experimental traction', the 'windowed traction' and the 'Fourier-series traction' approaches, respectively, compared to the case where the tractions are known, at high noise level. Computation time using the fast algorithm [30] was in each case very close to the value of 2.5 $\mathrm{s}$ required by the known-traction reconstruction algorithm for a nearly thousand degree of freedom problem and $1000 \times 1000$ pixel input strain fields ${ }^{3}$. All three approaches can also be applied to the case of non-rectangular domains. In view of the superior noise performance of the 'windowed traction' approach, this is recommended as the best of the three methods investigated here. When this approach was applied to experimental data from a circular disc under diametral compression loading, reasonable agreement between theory and experiment for both the stiffness and traction distributions was achieved.

\footnotetext{
${ }^{3}$ The computation was implemented on an Intel ${ }^{\circledR}$ Core $^{\mathrm{TM}}$ i7 CPU $2.79 \mathrm{GHz}$ desktop PC with $8 \mathrm{~GB}$ of memory, using the MATLAB programming language.
} 


\section{References}

1. Barbone P.E. and Oberai A.A. (2007) Elastic modulus imaging: Some exact solutions of the compressible elastography inverse problem. Phys Med Biol 52, 1577-1593.

2. Albocher U., Oberai A.A., Barbone P.E. and Harari I. (2009) Adjoint-weighted equation for inverse problems of incompressible plane-stress elasticity. Computer Methods in Applied Mechanics and Engineering 198, 2412-2420.

3. Doyley M.M., Meaney P.M. and Bamber J.C. (2000) Evaluation of an iterative reconstruction method for quantitative elastography. Phys Med Biol 45, 1521-1540.

4. Xu B., Wu Z.S., Chen G.D. and Yokoyania K. (2004) Direct identification of structural parameters from dynamic responses with neural networks. Eng Appl Artif Intel 17, 931-943.

5. Avril S., Bonnet M., Bretelle A.-S., Grédiac M., Hild F., Ienny P., Latourte F., Lemosse D., Pagano S., Pagnacco E. and Pierron F. (2008) Overview of identification methods of mechanical parameters based on full-field measurements. Exp Mech 48, 381-402.

6. Pierron F. and Grédiac M. (2012) The Virtual Fields Method: extracting constitutive mechanical parameters from full-field deformation measurements Springer New York.

7. Pourgholi R., Ebrahimian M., Emamjome M. and Reihani P. (2007) A numerical solution of an inverse parabolic problem with unknown boundary conditions. Appl Math Comput 189, 228-234.

8. Dennis B.H., Dulikravich G.S. and Yoshimura S. (2004) A finite element formulation for the determination of unknown boundary conditions for three-dimensional steady thermoelastic problems. $J$ Heat Transf 126, 110-118.

9. Nakajima M., Hayami K., Terao J., Watanabe S. and Ando S. (2000) Identification of tractions based on displacement observations at interior points. In: Inverse Problems in Engineering Mechanics II, M. Tanaka and G.S. Dulikravich (Eds). Elsevier Science Ltd, Oxford: 119-128.

10. Martin T.J., Halderman J.D. and Dulikravich G.S. (1995) An inverse method for finding unknown surface tractions and deformations in elastostatics. Comput Struct 56, 825835.

11. Sun Y., Ma F. and Zhang D. (2014) An integral equations method combined minimum norm solution for 3D elastostatics Cauchy problem. Computer Methods in Applied Mechanics and Engineering 271, 231-252.

12. Arai M., Nishida T. and Adachi T. (2000) Identification of dynamic pressure distribution applied to the elastic thin plate. In: Inverse Problems in Engineering Mechanics II, M. Tanaka and G.S. Dulikravich (Eds). Elsevier Science Ltd, Oxford: 129-138.

13. Avril S., Huntley J.M., Pierron F. and Steele D.D. (2008) 3D heterogeneous stiffness reconstruction using MRI and the virtual fields method. Exp Mech 48, 479-494.

14. Pierron F., Bayly P.V. and Namani R. (2010) Application of the virtual fields method to magnetic reasonance elastography data. Proc. SEM Annual Conf and Expo on Expt and Appl Mechanics, Indianapolis, Indiana, USA.

15. Devivier C., Pierron F. and Wisnom M.R. (2013) Impact damage detection in composite plates using deflectometry and the Virtual Fields Method. Compos A: Appl Sci Manuf 48, 201-218. 
16. Nguyen T.T., Huntley J.M., Ashcroft I.A., Ruiz P.D. and Pierron F. (2014) A Fourierseries-based virtual fields method for the identification of 2-D stiffness distributions. Int $J$ Numer Meth Eng 98, 917-936.

17. Grediac M., Toussaint E. and Pierron F. (2002) Special virtual fields for the direct determination of material parameters with the virtual fields method. 1 - Principle and definition. Int J Solids Struct 39, 2691-2705.

18. Kim J.H., Pierron F., Wisnom M.R. and Syed-Muhamad K. (2007) Identification of the local stiffness reduction of a damaged composite plate using the virtual fields method. Compos A: Appl Sci Manuf 38, 2065-2075.

19. Kim J.-H. (2008) Identification of the local stiffness reduction of a damaged composite plate using the virtual fields method. PhD, Arts et Metiers ParisTech.

20. Le Louëdec G., Sutton M.A. and Pierron F. (2011) Local elasto-plastic identification of the behaviour of friction stir welds with the virtual fields method. Appl Mech Mater 70, $135-140$.

21. Sutton M.A., Yan J.H., Avril S., Pierron F. and Adeeb S.M. (2008) Identification of heterogeneous constitutive parameters in a welded specimen: Uniform stress and virtual fields methods for material property estimation. Exp Mech 48, 451-464.

22. Tattoli F., Pierron F., Rotinat R., Casavola C. and Pappalettere C. (2010) Full-field strain measurement on titanium welds and local elasto-plastic identification with the virtual fields method. Proc. Inter Conf on Adv in Mater and Process Technol 860-865.

23. Hibbeler R.C. and Fan S.C. (2004) Statics and mechanics of materials. SI edn. Pearson Prentice Hall.

24. Hartley R. and Zisserman A. (2004) Multiple view geometry in computer vision Cambridge University Press.

25. Campbell S.L. and Meyer C.D. (1991) Generalized inverses of linear transformations Dover, New York.

26. Nguyen T.T. (2013) The Fourier virtual fields method for the identification of material property distributions. $\mathrm{PhD}$, Loughborough University.

27. Avril S., Ferrier E., Vautrin A., Hamelin P. and Surrel Y. (2004) A full-field optical method for the experimental analysis of reinforced concrete beams repaired with composites. Compos A: Appl Sci Manuf 35, 873-884.

28. Timoshenko S.P. and Goodier J.N. (1970) Theory of elasticity McGraw Hill, New York.

29. Procopio A.T., Zavaliangos A. and Cunningham J.C. (2003) Analysis of the diametrical compression test and the applicability to plastically deforming materials. J Mater Sci 38, 3629-3639.

30. Bracewell R.N. (1986) The Fourier transform and its applications McGraw-Hill. 
Strain

Page 22 of 33

Table 1: Traction components for particular values of angle $\theta$

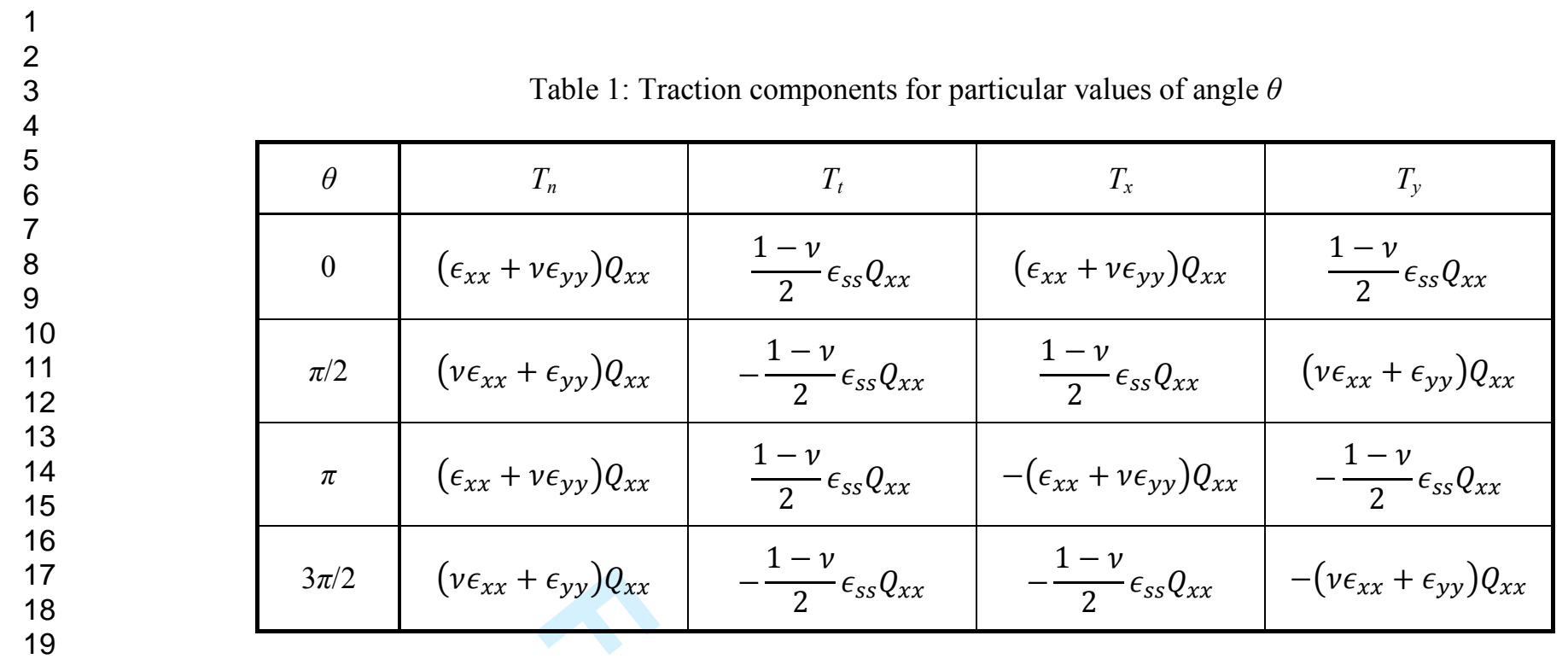

22 
Traction components on an arbitrary edge of a 2-D domain inclined an angle $\theta$ about the vertical direction. $45 \times 27 \mathrm{~mm}(300 \times 300 \mathrm{DPI})$ 


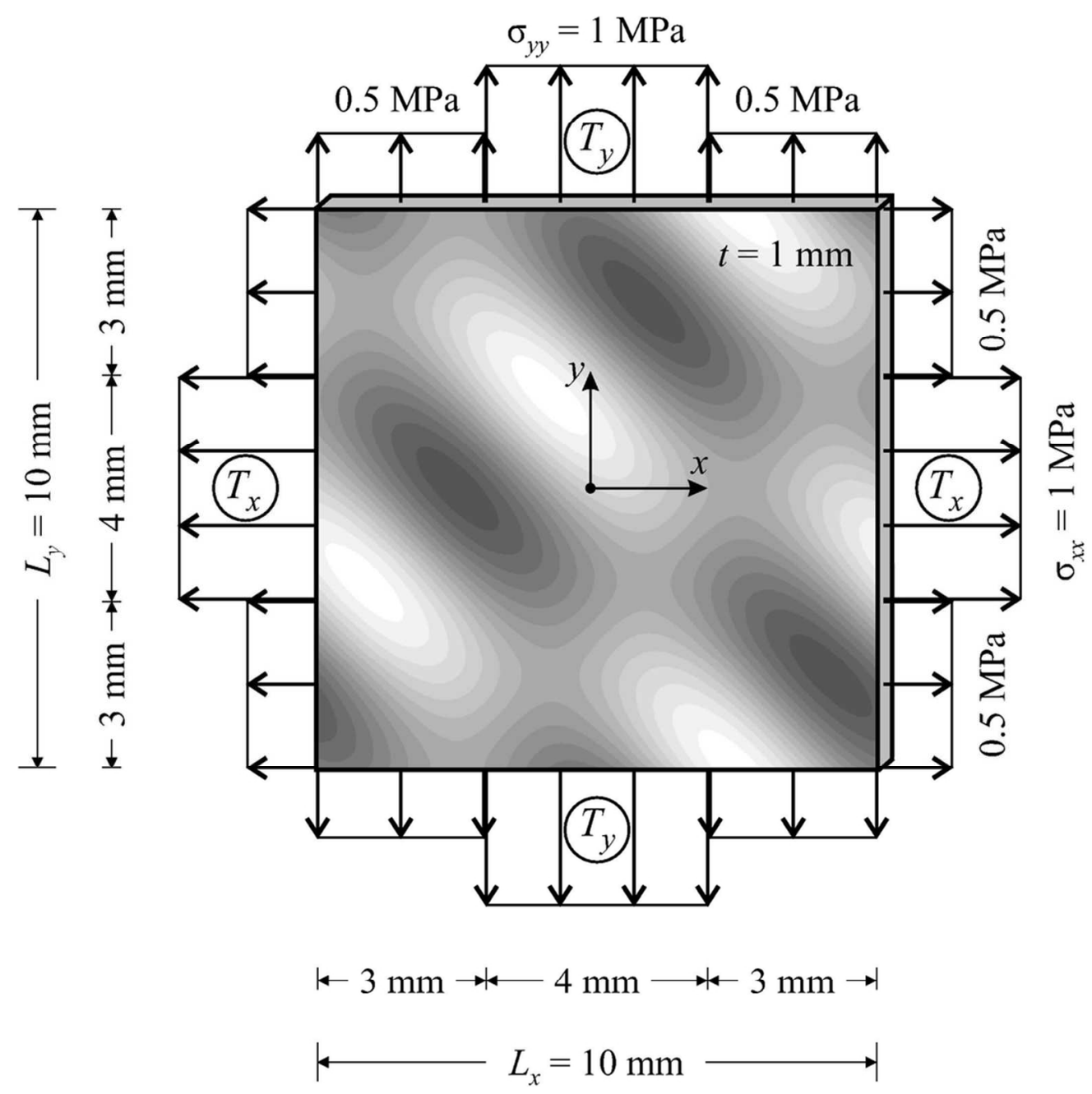

A square plate with an 'egg-box' stiffness distribution subject to biaxial loading with stepped stress profile in all directions. The origin of the coordinate system is at the centre of the plate. $91 \times 91 \mathrm{~mm}(300 \times 300 \mathrm{DPI})$ 
(a)

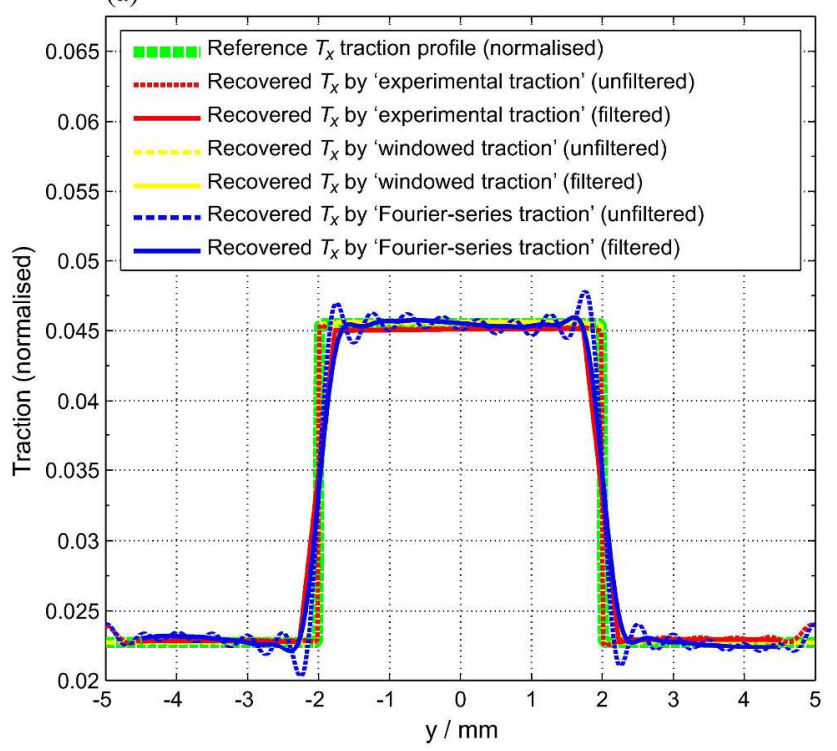

(b)

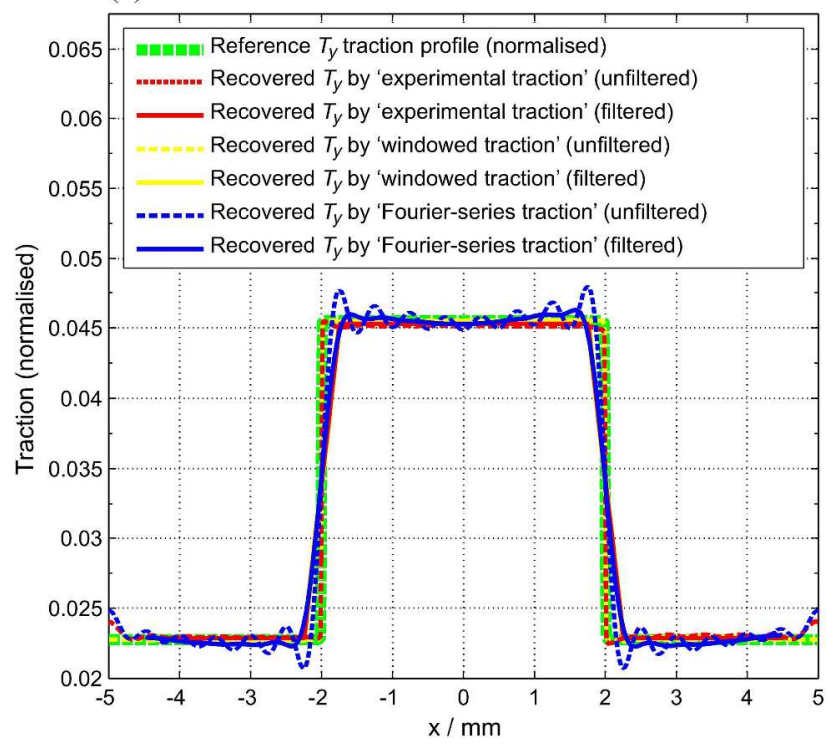

Reconstruction of traction profiles (a) $T_{x}$ and (b) $T_{y}$ applied on an 'egg-box' stiffness plate. The reconstruction is based on the resulting stiffness maps shown in Figure 3 recovered by three different approaches.

$202 \times 389 \mathrm{~mm}(300 \times 300$ DPI $)$ 


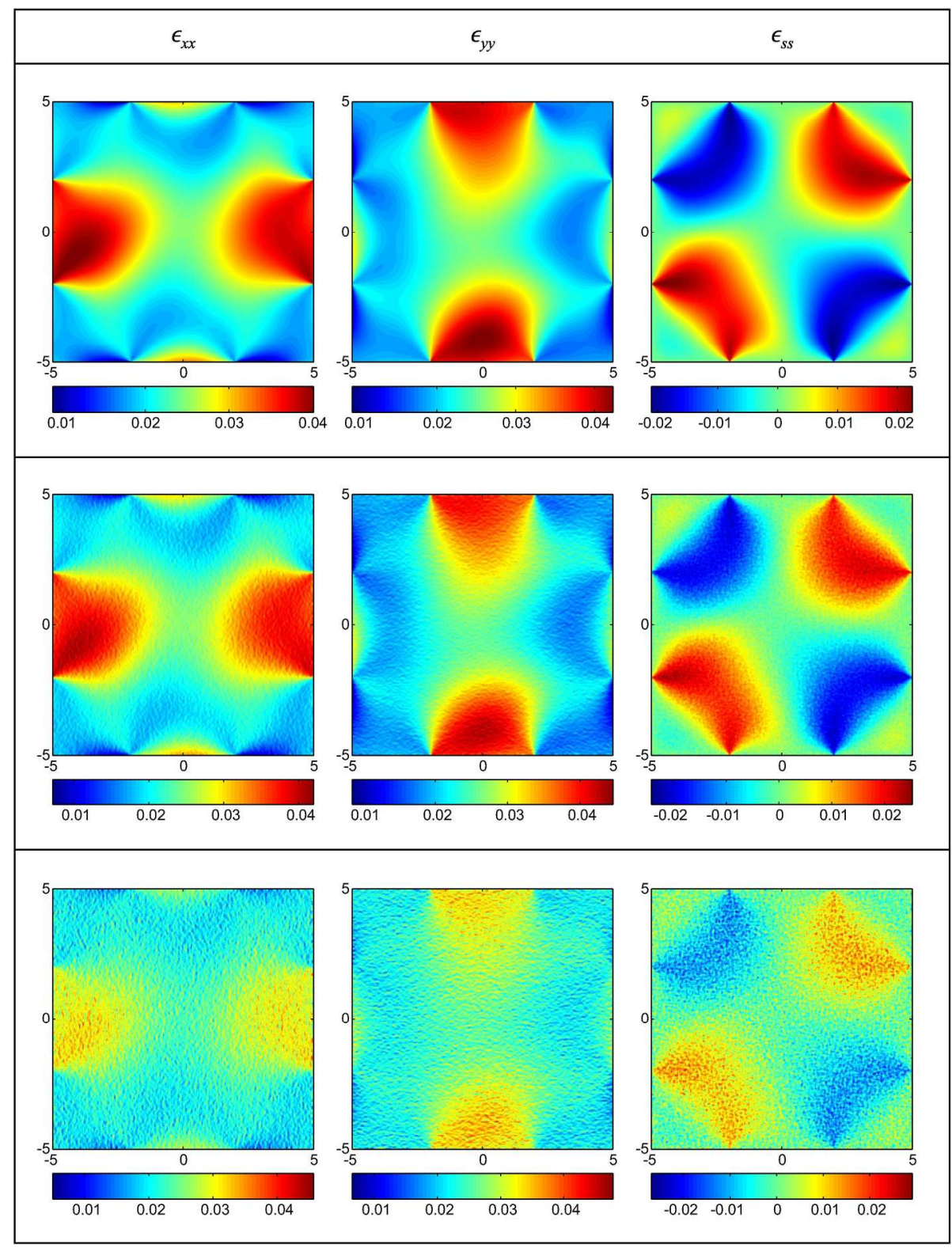

Numerical strain fields of an 'egg-box' pattern obtained from a forward finite element analysis, differentiated from displacement fields with a gradient kernel size of $13 \times 13$ pixels, with and without the presence of noise. The three strain fields, $\epsilon_{x x}, \epsilon_{y y}$ and $\epsilon_{s s}$, are shown respectively in the left-hand, middle and right-hand columns of the figure. Figures in the top row are noise free, in the middle row are with displacement noise level $\sigma=5 \times 10^{-3} \mathrm{~mm}$ (medium noise level) and in bottom row with $\sigma=10^{-2} \mathrm{~mm}$ (high noise level). $204 \times 269 \mathrm{~mm}(300 \times 300 \mathrm{DPI})$ 
Reconstruction of a normalised 'egg-box' stiffness pattern of a plate under unknown boundary tractions, with noisy input strain fields from a finite element model. Top, middle and bottom rows show respectively results from the 'experimental traction', 'windowed traction' and 'Fourier-series traction' approaches. Left, middle and right columns show respectively the reconstructed stiffness patterns, the distributions after ripple removal, and the errors in recovered stiffness with respect to the normalised reference stiffness pattern. An extra number of cosine/ sine terms in the traction Fourier series of the 'Fourier-series traction' approach is $K$ $=20$. Displacement-gradient kernel size $=13 \times 13$ pixels, medium noise level throughout. $195 \times 246 \mathrm{~mm}(300 \times 300 \mathrm{DPI})$ 
(a)

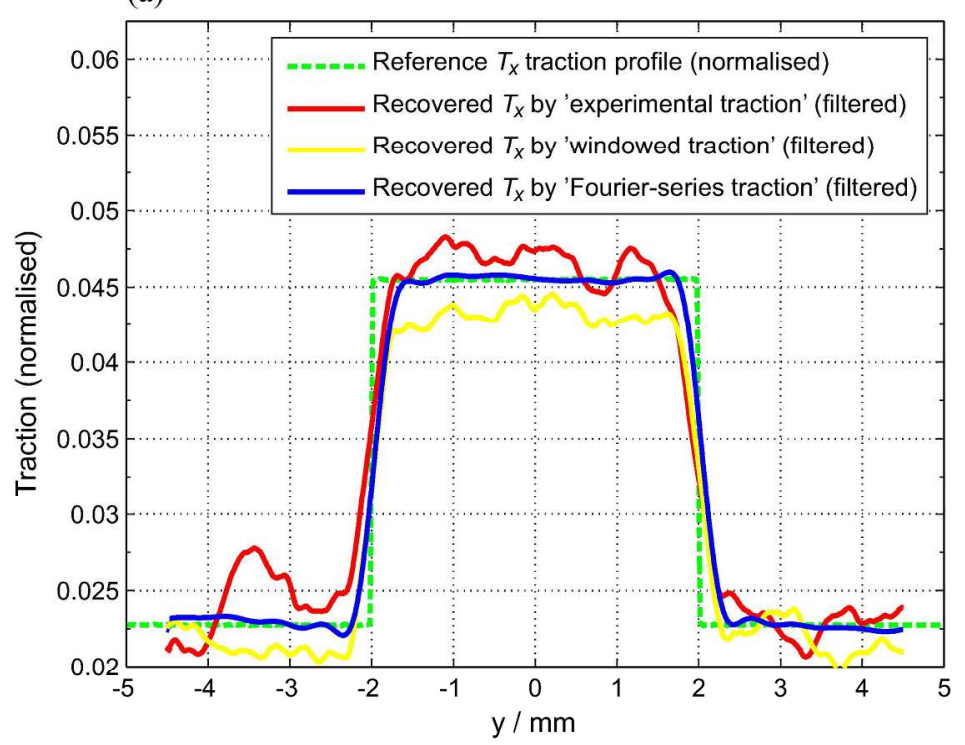

(b)

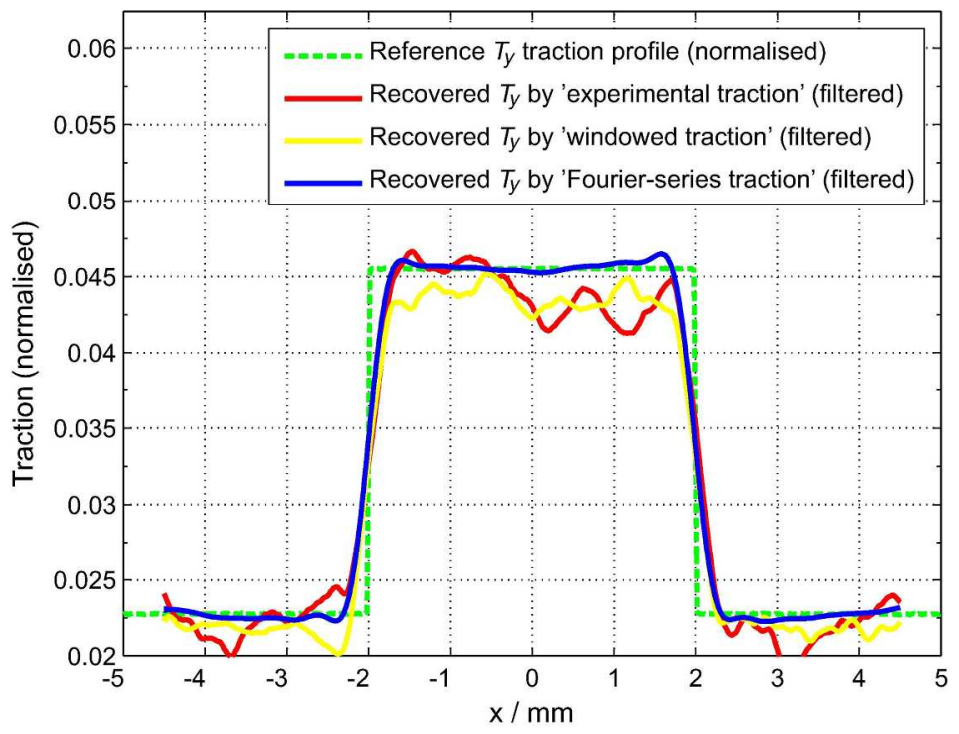

Reconstruction of traction profiles (a) $T_{x}$ and (b) $T_{y}$ applied to a plate with 'egg-box' stiffness distribution, in the presence of noise and after data smoothing/filtering. The reconstruction is based on the resulting stiffness maps shown in Figure 6 recovered by three different approaches.

$175 \times 292 \mathrm{~mm}(300 \times 300$ DPI $)$ 

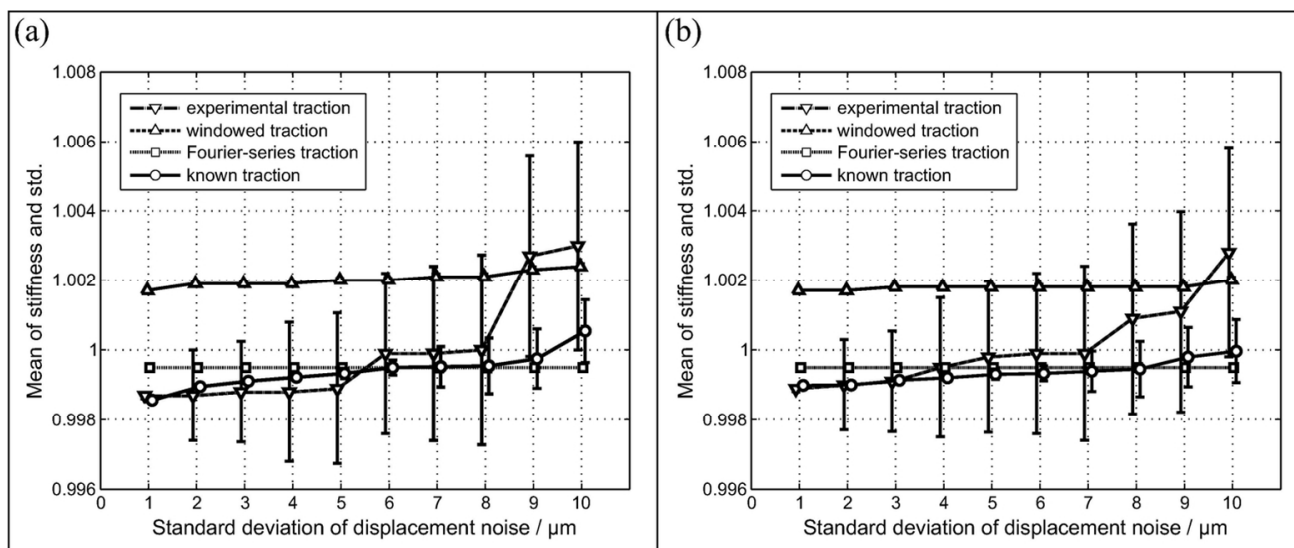

(c)

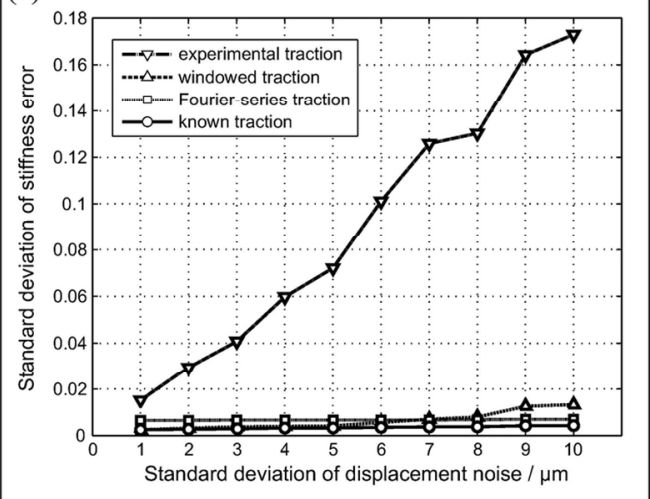

(d)

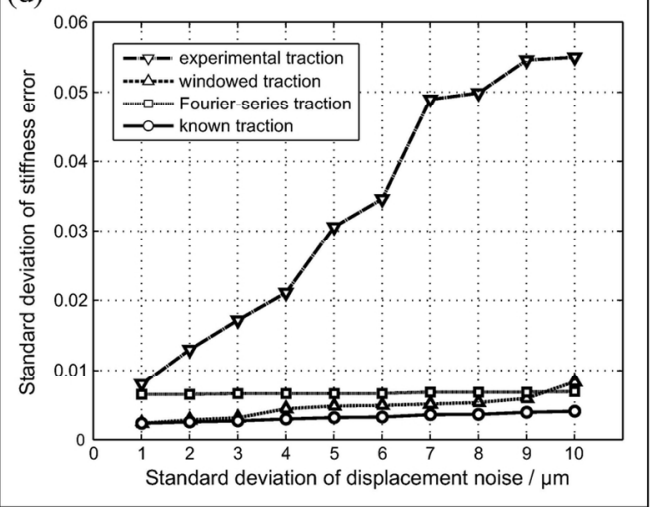

Mean error $(a, b)$ and standard deviation of the error $(c, d)$ in the recovered stiffness distribution for the 2-D 'egg-box' stiffness pattern. The differentiation from noisy displacement fields was carried out with kernel sizes of $7 \times 7(a, c)$ and $13 \times 13(b, d)$ pixels. In (a) and (b), markers indicate the mean, and error bars the standard deviation in the mean. $129 \times 107 \mathrm{~mm}(300 \times 300 \mathrm{DPI})$ 


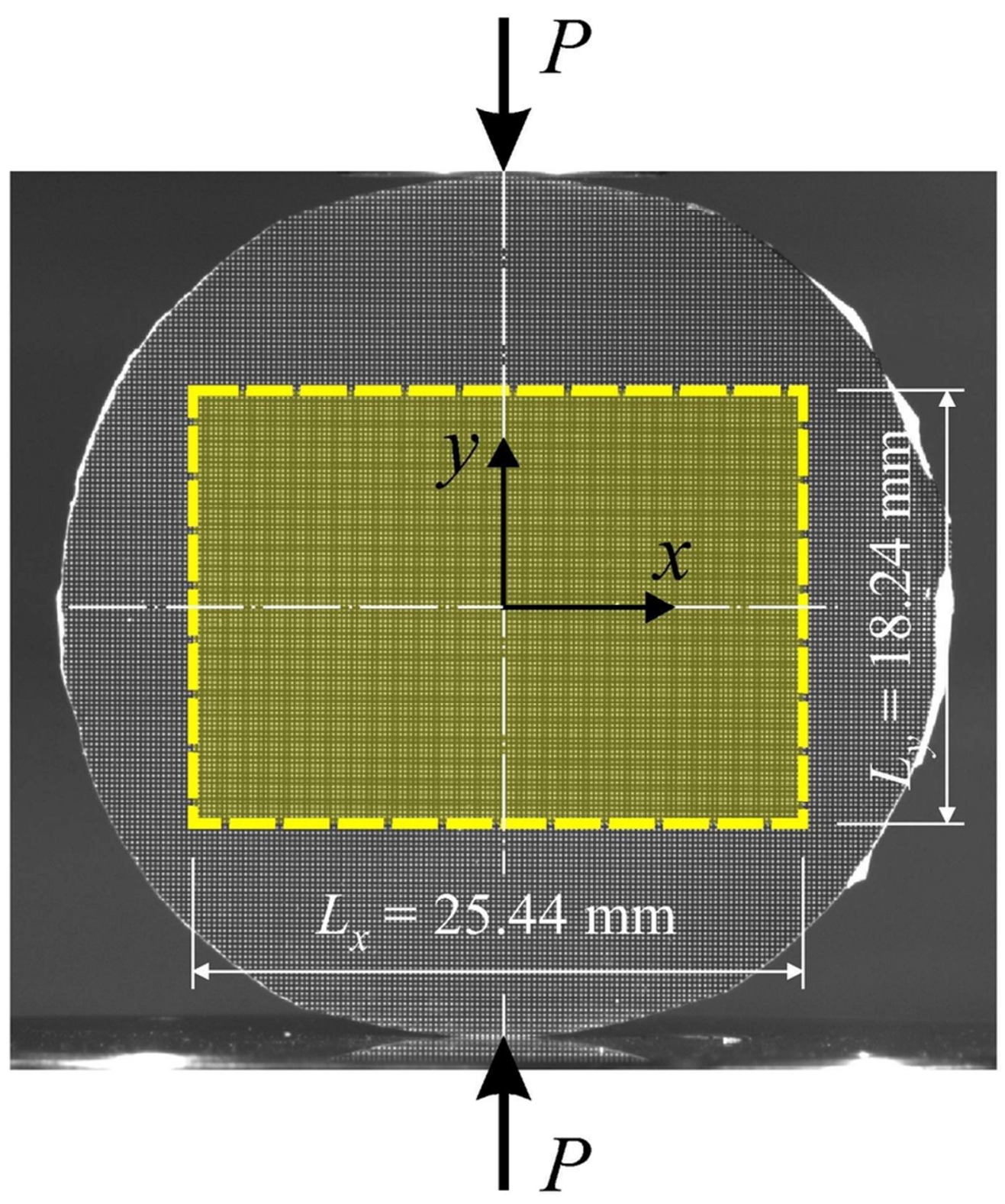

Schematic of an aluminium circular disc of diameter $36 \mathrm{~mm}$ under compressive load with a highlighted region of interest (ROI) of size $L_{x} \times L_{y}=25.44 \times 18.24 \mathrm{~mm}^{2}$ within which the strain fields are extracted. $69 \times 82 \mathrm{~mm}(300 \times 300 \mathrm{DPI})$ 
( $a, b, c)$ Full-field experimental strain field components of a circular disc under diametral compression loading. ( $d, e, f)$ Experimental strain field components within the ROI. $(g, h, i)$ Corresponding theoretical strain field components computed from the Hertz contact theory. In each sub-figure, the horizontal and vertical axes represent respectively $x / D$ and $y / D$. The columns from left to right contain the components $\epsilon_{x x}$, $\epsilon_{y y}$ and $\epsilon_{s s}$, respectively. $178 \times 205 \mathrm{~mm}(300 \times 300 \mathrm{DPI})$

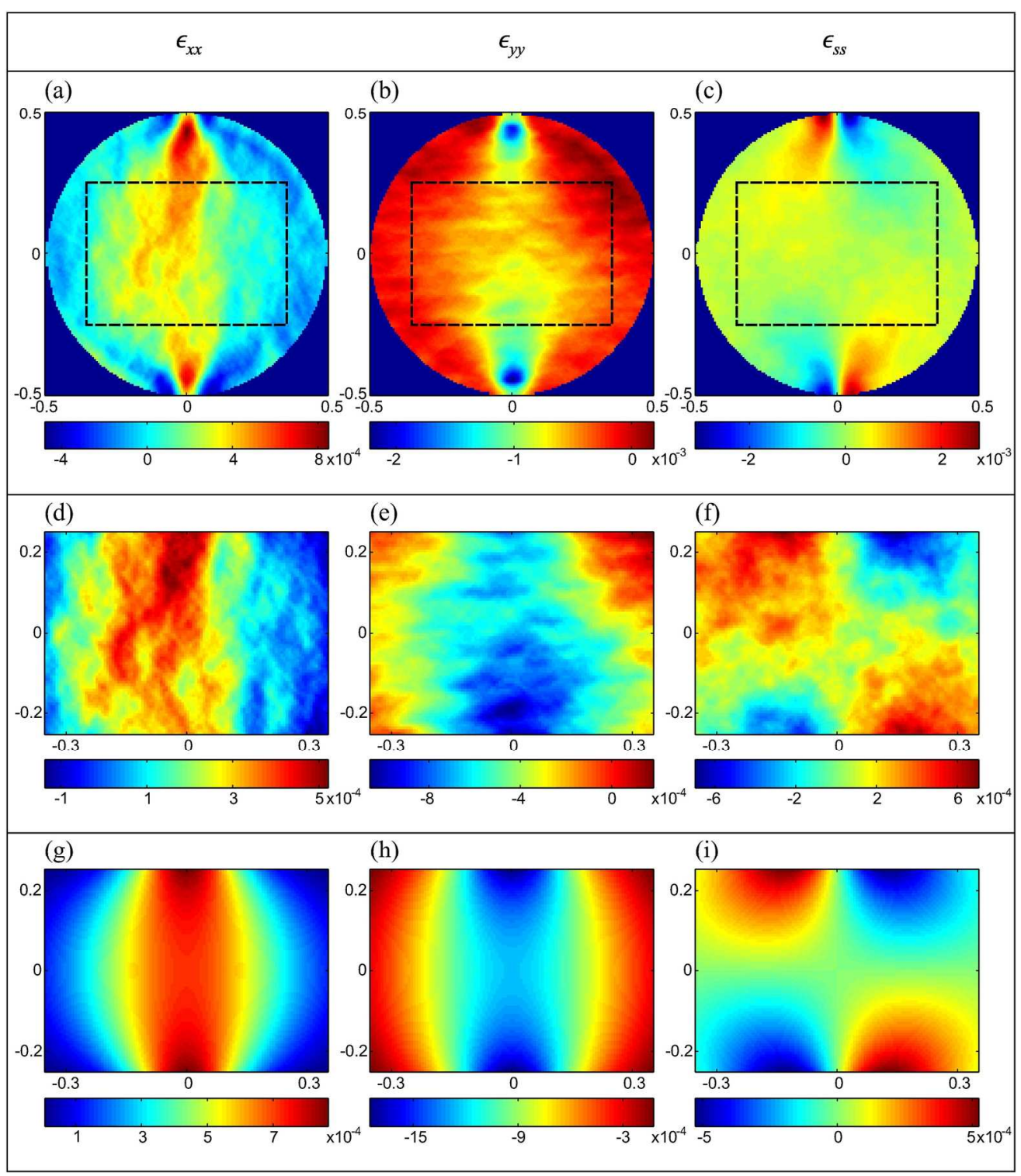



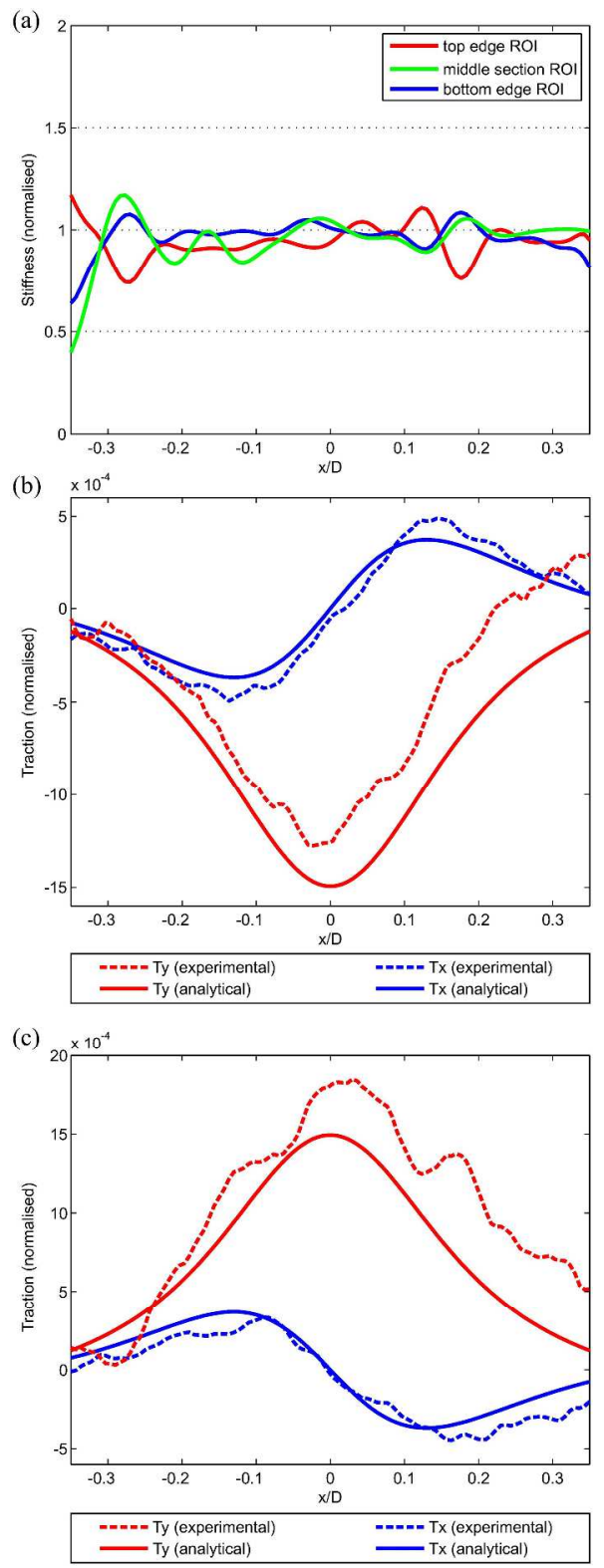

(a) Stiffness profiles at particular cross sections reconstructed by the F-VFM. (b), (c) Recovered nondimensional traction profiles $T_{x}$ (in blue) and $T_{y}$ (in red) on (b) the top edge and (c) the bottom edge of the ROI compared to their counterparts from Hertz contact theory. $241 \times 646 \mathrm{~mm}(300 \times 300 \mathrm{DPI})$ 